\title{
Eight Years of Data on Chlamydia trachomatis Epidemiology in Germany
}

Sandra Dudareva', Viviane Bremer', Matthias an der Heiden', Kerstin Dehmel', Andrea Sailer', Klaus Jansen'

\section{Background \& aim}

- Chlamydia trachomatis (CT) infections are not reportable in Germany and limited data on prevalence available

- Women and men are tested due to symptoms

- Women are offered screening

- Screening in pregnancy (since 1995)

- Screening for women $<25$ years (since 2008)

$>$ Tests covered by the state health insurance, however physicians not remunerated for counselling

Aim:

We analysed CT-data to develop recommendations for prevention

\section{Methods}

- 2010: voluntary laboratory-based sentinel surveillance implemented

- Collection of retrospective (starting 2008) and prospective data on CT tests together with results and information on sex, age, and test reason

- Calculation of proportion of positive tests by sex, age-group in years and test reason, comparison by $\mathrm{Chi}^{2}$ test

- Estimation of proportion of target population $<25$ years reached by screening for women

- Trend estimation of proportion of positive tests by sex, age-group and test reason with multivariable Poisson regression

\section{Results}

Data 2008-2016 (September 2017)

- 5,706,455 tests from 25 laboratories from allover Germany

- $93 \%$ from women

Proportion of CT-Tests by test reason (2014-2016)

- $24 \%$ Screening in pregnancy

- $41 \%$ Screening $<25$ years

- $26 \%$ Test due to symptoms

Coverage of Screening for women $<25$ years

- Since 2011 12\% yearly (2008 8\%)

Figure 1: Proportion of CT-tests by sex and age group (2014-2016)

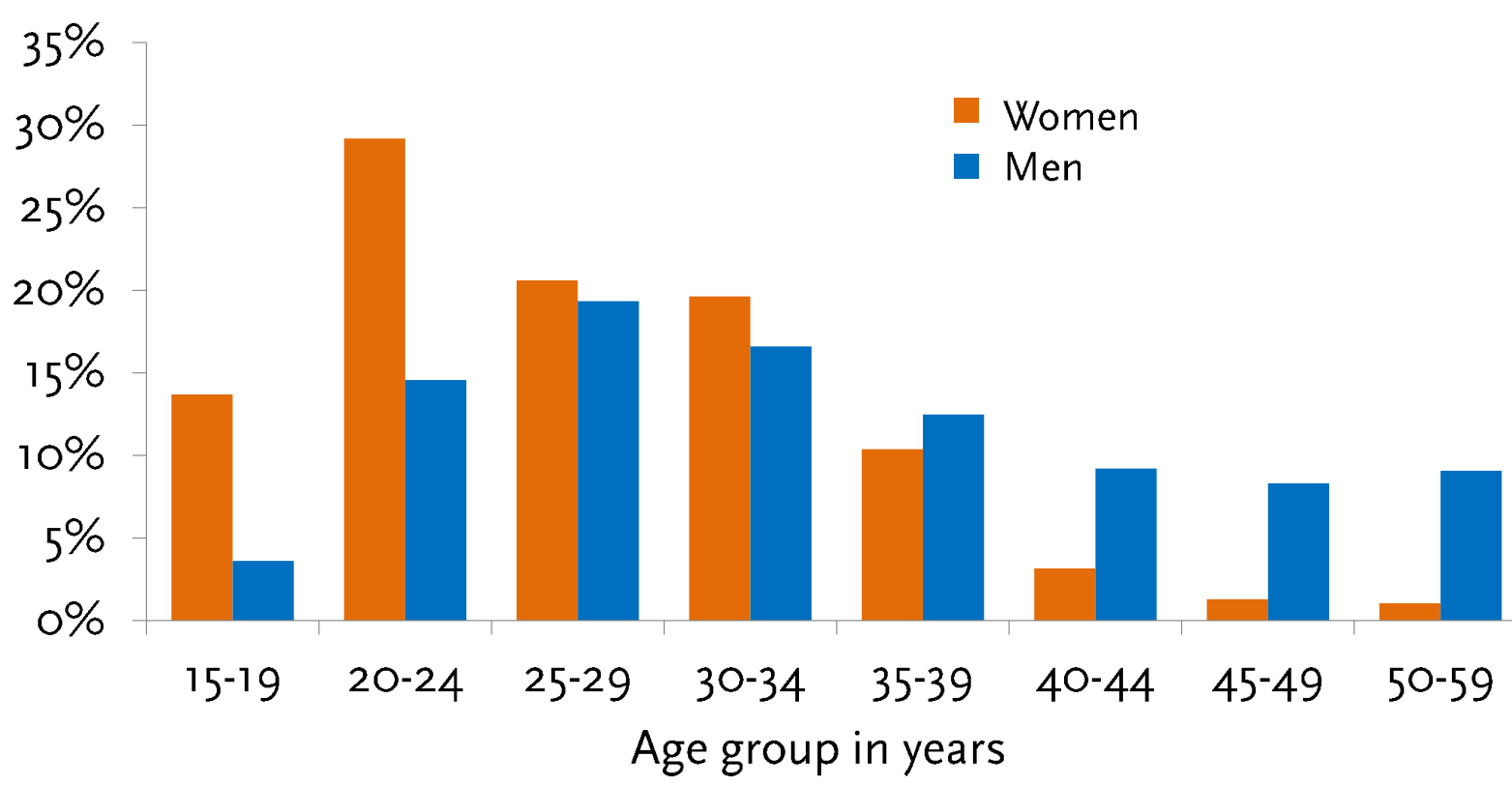

Figure 2: Proportion of positive CT-tests by sex and age-group (2014-2016)

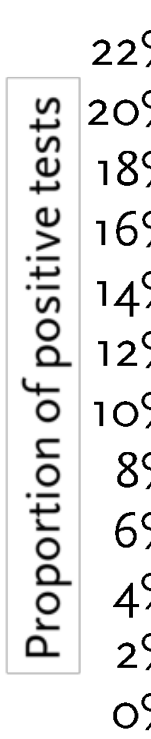

$22 \%$

出 $20 \%$

$\stackrel{\Perp}{ \pm} 18 \%$

$16 \%$

$14 \%$

․ $12 \%$

$10 \%$

$8 \%$

$6 \%$
$4 \%$

$4 \%$ ०\%

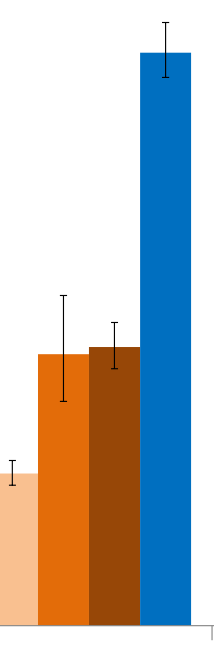

$15-19$

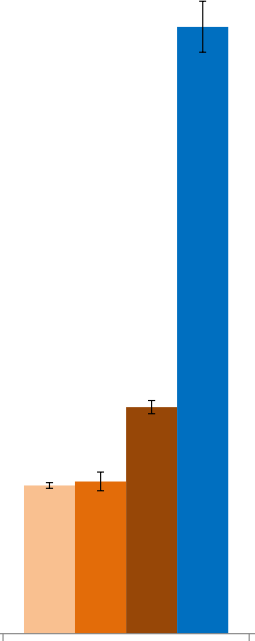

$20-24$
Women: Screening <25 years

- Women: Screening in pregnancy

- Women: Test due to symptoms

- Men: Test due to symptoms

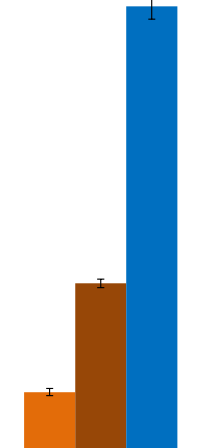

$25-29$

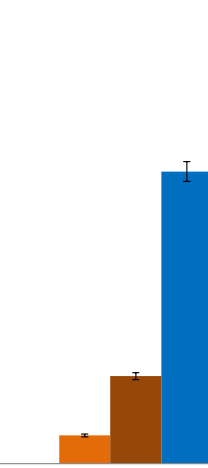

30-34

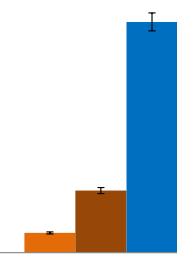

$35-39$

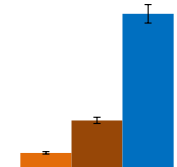

$40+$
Age group in years

\section{Conclusions}

- Proportion of positive CT-tests:

- High among young women and men

- Slight decrease among 15-29 years old women and men over 25 years

- Increase among 15-19 years old men

- Screening for women <25 years coverage in Germany:

- Coverage insufficient

- Did not increase substantially in the last years
Figure 3: Proportion of positive CT-tests among women by age group over time (2008-2016)

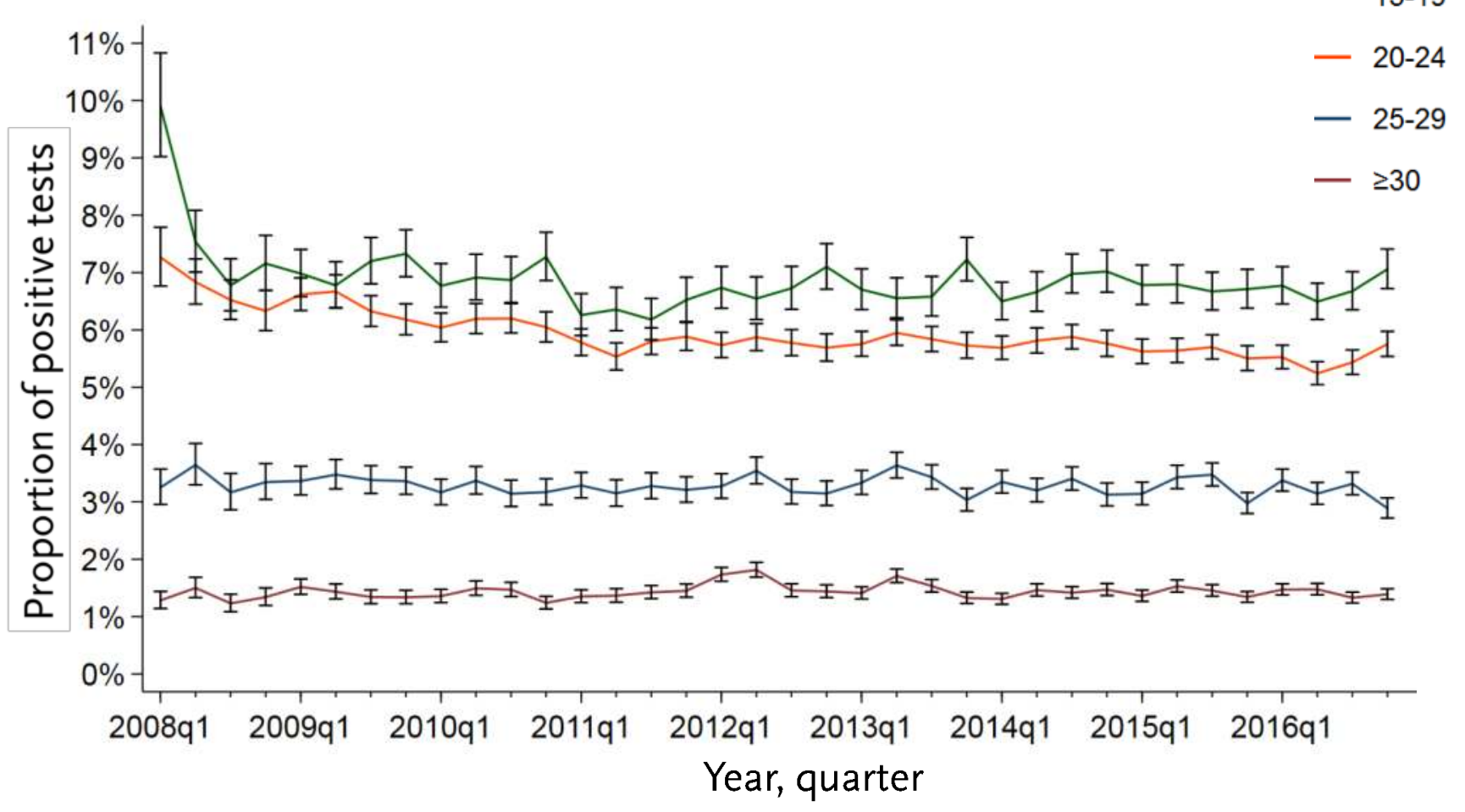

- 15-19 years -0.5\%/per year

- $20-24$ years $-1,9 \% /$ per year

- $25-29$ years $-0,5 \% /$ per year

Figure 3: Proportion of positive CT-tests among men by $\quad-15-24$ age group over time (2008-2016)

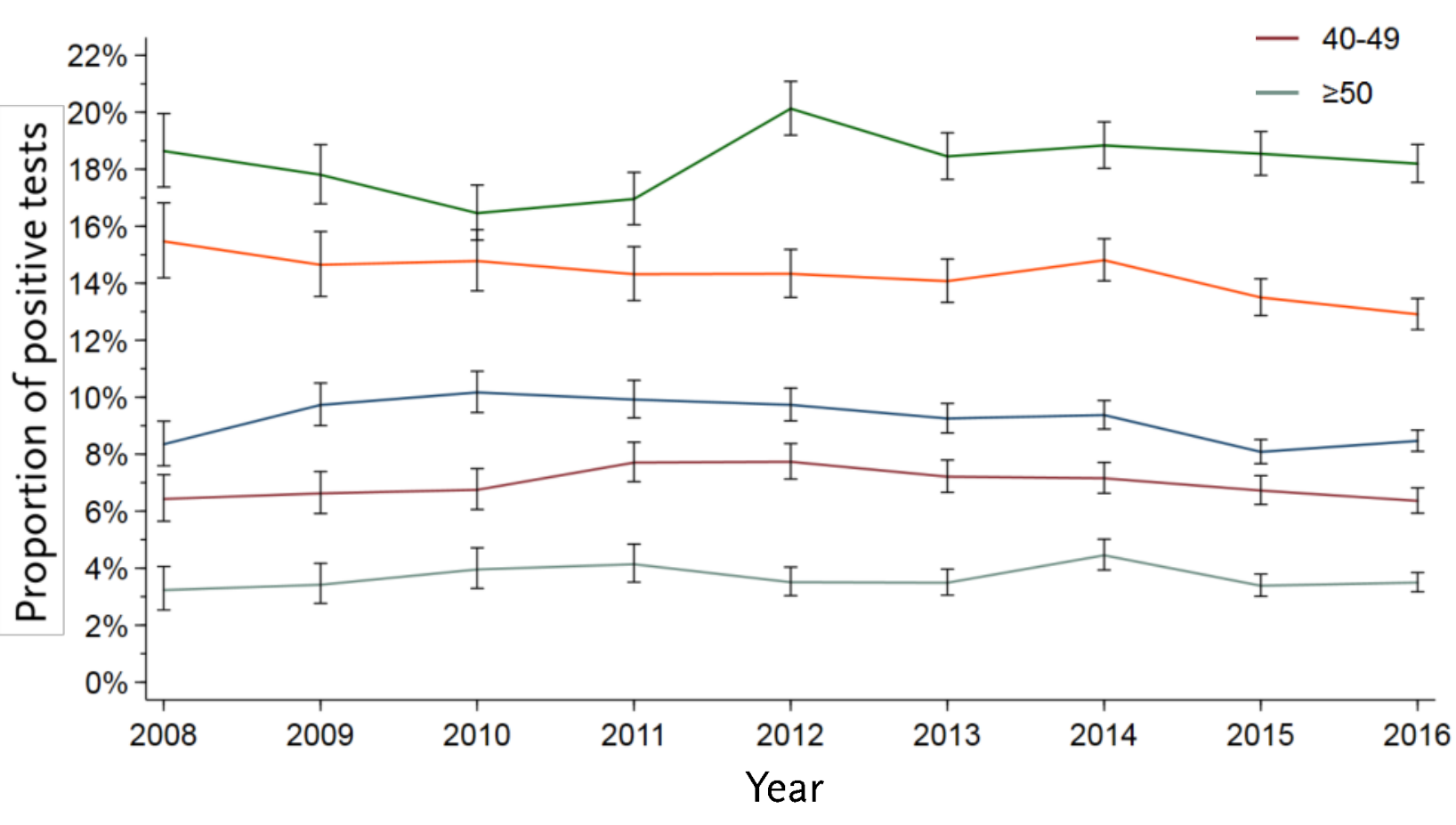

- $15-19$ years $+3.8 \% /$ per year $\quad-25-29$ years $-1,9 \% /$ per year

\section{Recommendations}

- Awareness campaigns for young women and men should be implemented

- Risk based testing for CT should be considered

- Screening for women <25 years among should be promoted

- Target population $\longrightarrow$ awareness campaigns

- Physicians $\longrightarrow$ remuneration for counselling

1 HIV/AIDS, STI and Bloodborne Infections Unit, Department for Infectious Disease Epidemiology, Robert Koch Institute, Berlin, Germany 